\title{
Intelligent Call Admission Control Using Fuzzy Logic in Wireless Networks
}

\author{
Yufeng Ma, Xiulin Hu, Yunyu Zhang, and Yimei Shi
}

\begin{abstract}
Scarcity of the spectrum resource and mobility of users make Quality-of-Service $(\mathrm{QoS})$ provision a critical issue in wireless networks. This paper presents a fuzzy call admission control scheme to meet the requirement of the QoS. It searches automatically the optimal number of the guard channels in a base station to make an effective use of resource and guarantee the QoS provision. Simulation compares the proposed fuzzy scheme with an adaptive channel reservation scheme. Simulation results show that fuzzy scheme has a better robust performance in terms of call dropping probability, call blocking probability, and channel utilization.
\end{abstract}

\section{INTRODUCTION}

$\mathrm{I}$ the present and the next generation wireless networks, cellular system is still a major part in telecommunication infrastructure. Cellular system exploits frequency reuse to achieve high capacity by limiting the coverage of each base station within a small geographic area called a cell. When the users move from one cell to another, handoff operation will occur. Mobile users may change cells many times during the lifetime of their connections. Specially, in future micro/pico-cellular architecture, handoff operation may occur more frequently than in present macro-cellular architecture. Since the user's itinerary and the availability of resources in various cells is not known in advance, it makes QoS provision a critical issue in cellular radio systems.

Call Admission Control (CAC) is one of the important

Manuscript received September 14, 2004.

Yufeng Ma is with the Department of Electronic and Information, Huazhong University of Science and Technology, Wuhan, Postal Code 430074, China (Telephone: (0086)27-83669893; e-mail: 050904@ 163.com).

Xiulin $\mathrm{Hu}$ is with the Department of Electronic and Information, Huazhong University of Science and Technology, Wuhan, Postal Code 430074, China (e-mail: hxlin@ public.wh.hb.cn).

Yunyu Zhang is with the Department of Electronic and Information, Huazhong University of Science and Technology, Wuhan, Postal Code 430074, China (e-mail: hxlin@ public.wh.hb.cn).

Yimei Shi is in 188 Chunjiu Road, 393 Yishashi Garden, Songjiang District, Shanghai, Postal Code 201600, China (e-mail: shiyimei2008@ 163.com). mechanisms in guarantying the QoS. It can be defined as the procedure of deciding whether or not to accept a new connection. If the network can not meet the connection need, the connection request will be denied. In cellular wireless networks, one important parameter of the QoS is call blocking probability (CBP), which indicates the likelihood of the new connection being denied. The other important parameter is call dropping probability (CDP), which expresses the likelihood of the existing connection being denied during handoff process due to insufficient resource in target cell. From the user's point of view, having a call abruptly terminated in the duration of the connection is more annoying than being blocked occasionally on a new call attempt. It is acceptable to give higher priority to handoff call. The methods for prioritizing handoff are the guard channel and queuing of handoff requests. With the guard channel method, a fraction of the total available channels in a cell is reserved exclusively for handoff requests from ongoing calls which may be handed off into the cell. The fixed reservation strategy wastes valuable spectrum resource while the dynamic reservation strategy can offer efficient spectrum utilization by minimizing number of the required guard channels.

In the present, there are many schemes about call admission control aiming at keeping the CDP and CBP low while maximizing the resource utilization to meet the system demand at the same time [1-6]. When the CDP decreases, the CBP will increase accordingly. So, it is hard to guarantee the minimum CDP and the minimum CBP at the same time. Minimizing the CDP is one of the main goals of QoS provisioning in wireless networks.

The main contribution of this paper is to propose an intelligent call admission control scheme based on fuzzy logic for cellular wireless networks. The scheme is built upon the concept of the guard channels. We design a fuzzy controller in terms of the important QoS target. The scheme adjusts the number of the guard channels to its optimum in time according to the CDP and current number of the guard channel. It tries to make an effective use of resource and keep the CDP and CBP low at the same time.

The remainder of this paper is organized as follows. Section 2 analyzes the method and model for call admission control. Section 3 gives the details of the proposed fuzzy call 
admission control scheme. Section 4 runs simulation to compare the fuzzy scheme with an adaptive channel reservation scheme. Then, it discusses the simulation results. Finally, section 5 gives conclusion of the paper.

\section{CAC METHOD AND MODEL ANALYSIS}

The total number of available channels (denoted by $\mathrm{C}$ ) in a cell consists of two parts. One part (denoted by $\mathrm{C}_{h}$ ) is reserved exclusively for handoff calls. The remaining $C-C_{h}$ channels are shared by both new calls and handoff calls. A new call will be admitted into the network if the number of busy channels in the cell is less than $\mathrm{C}-\mathrm{C}_{\mathrm{h}}$ when the call is originated. A handoff request will be admitted if the number of busy channels in the target cell is less than C.

We assume that the arrivals of new call and handoff call are independent Poisson processes. The new call arrival rate is $\lambda_{n}$ and the handoff call arrival rate is $\lambda_{h}$. Channel holding times are assumed to follow a negative exponential distribution with mean $1 / \mu$. For a cell capacity of $\mathrm{C}$, queuing model is considered as $\mathrm{M} / \mathrm{M} / \mathrm{C}$ in which the $\mathrm{C}$ available channels in the cell represented as $\mathrm{C}$ servers. The system state space is a finite set $\mathrm{E}=\{0,1,2, \ldots, \mathrm{C}\}$. The state transition diagram is shown in Fig.1.
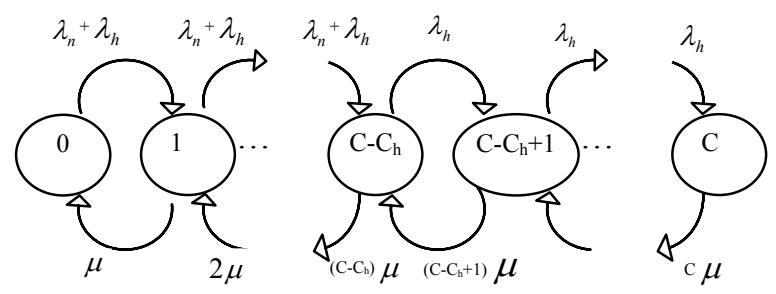

Fig.1. State-transition diagram

Let $P_{j}$ represent the steady-state probability that the base station is in state $\mathrm{j}$. During the analysis of birth-death process, the probability $\mathrm{P}_{\mathrm{j}}$ can be obtained like that $[1,4]$ :

$$
\begin{aligned}
& P_{0}=\left[\sum_{k=0}^{C-C_{h}} \frac{\left(\lambda_{n}+\lambda_{h}\right)^{k}}{k ! \mu^{k}}+\sum_{k=C-C_{h}+1}^{C} \frac{\left(\lambda_{n}+\lambda_{h}\right)^{C-C_{h}} \lambda_{h}^{k-C+C_{h}}}{k ! \mu^{k}}\right]^{-1} \\
& P_{j}=\left\{\begin{array}{l}
\frac{\left(\lambda_{n}+\lambda_{h}\right)^{j}}{j ! \mu^{j}} P_{0}, 1 \leq j \leq C-C_{h} \\
\frac{\left(\lambda_{n}+\lambda_{h}\right)^{C-C_{h}} \lambda_{h}^{j-C+C_{h}}}{j ! \mu^{j}} P_{0}, C-C_{h}+1 \leq j \leq C
\end{array}\right.
\end{aligned}
$$

The new call will be blocked if the number of busy channels is more than $\mathrm{C}-\mathrm{C}_{\mathrm{h}}$. Hence

$$
\mathrm{CBP}=\sum_{j=C-C_{h}}^{C} P_{j} .
$$

The handoff request will be denied if the number of busy channels is equal to $\mathrm{C}$. Thus

$\mathrm{CDP}=\mathrm{P}_{\mathrm{C}}$.

\section{FUZZY LOGIC CALL ADMISSION CONTROL SCHEME}

In the guard channel method, the number of the guard channels is important to the performance of wireless network. It affects the QoS and resource utilization of the network. The proposed fuzzy scheme tries to adjust dynamically the number of guard channels to its optimum to meet the requirements of the QoS and resource utilization.

\section{A. Structure of Fuzzy Logic Controller (FLC)}

The concept of fuzzy set is an extension of classical set. For a classical set $\mathrm{X}$, an element may belong to set $\mathrm{X}$ or not. But for a fuzzy set, an element is related to a set by a membership function $\mu$. The membership function usually take on a value between 0 and 1. A FLC can provide algorithms which convert the linguistic control strategies based on intuition, heuristic learnings and export knowledge into an automatic control strategy. The FLC is made of fuzzifier, inference engine, Fuzzy Rule Base and defuzzifier. The structure of FLC is shown in Fig.2. This paper designs a FLC of two input parameters and one output parameter based on Mamdani fuzzy model. The input linguistic parameters of the FLC are set as call dropping probability (CDP) and number of the guard channels $\left(C_{h}\right)$. The output linguistic parameter is set as the tuning number of the guard channels $\left(\Delta \mathrm{C}_{\mathrm{h}}\right)$.

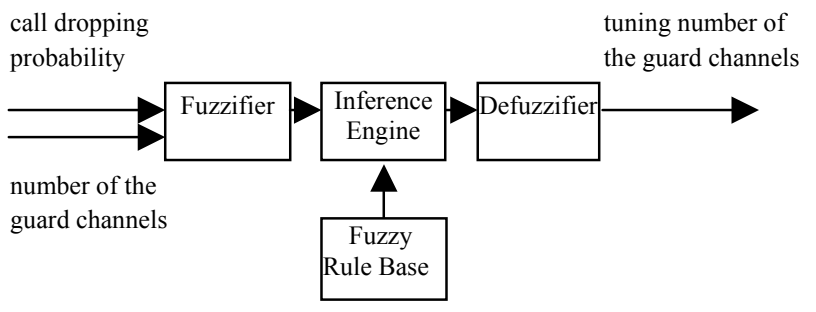

Fig.2. FLC structure

\section{B. Membership Functions}

We choose thresholds for CDP and number of the guard channels as 0.01 and $12 \%$ of the total number of channels in a cell respectively. The tuning number of the guard channels is chosen to vary within the range from $-12 \%$ to $+12 \%$ of the total channel capacity of a cell.

The term sets of CDP, $C_{h}$, and $\Delta C_{h}$ are defined as follows:

$\mathrm{T}(\mathrm{CDP})=\{\mathrm{Z}, \mathrm{VS}, \mathrm{S}, \mathrm{M}, \mathrm{B}, \mathrm{VB}\}$

$\mathrm{T}\left(\mathrm{C}_{\mathrm{h}}\right)=\{\mathrm{VS}, \mathrm{S}, \mathrm{M}, \mathrm{B}, \mathrm{VB}\}$

$\mathrm{T}\left(\Delta \mathrm{C}_{\mathrm{h}}\right)=\{\mathrm{NB}, \mathrm{NM}, \mathrm{NS}, \mathrm{NVS}, \mathrm{Z}, \mathrm{PVS}, \mathrm{PS}, \mathrm{PM}, \mathrm{PB}\}$

We choose triangular functions as membership functions because they are simple and practical. The membership functions for input and output linguistic parameters are shown in Fig.3, Fig.4, and Fig.5. 


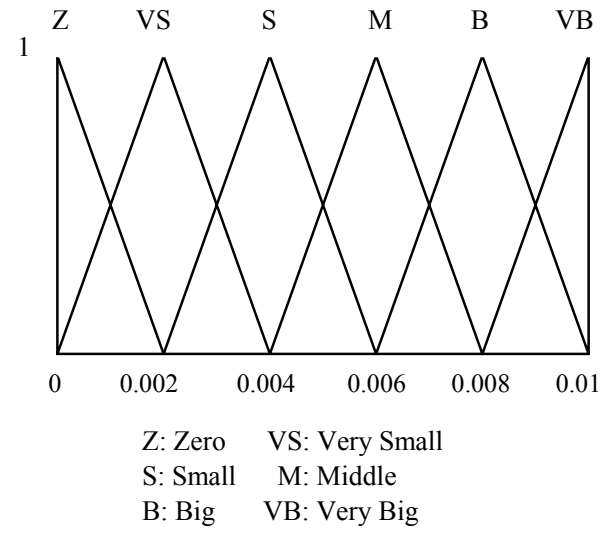

Fig.3. Membership functions for call dropping probability

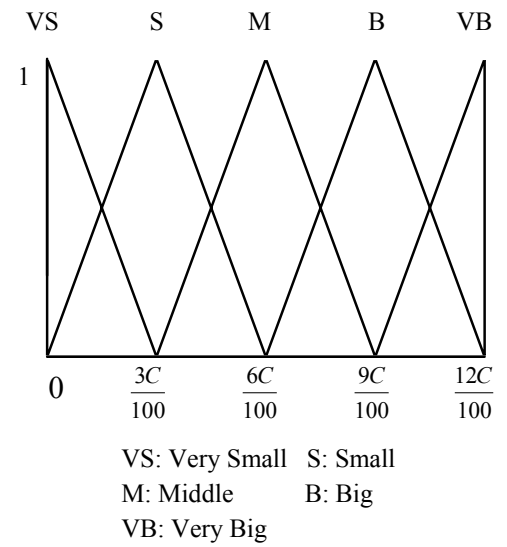

Fig.4. Membership functions for number of the guard channels

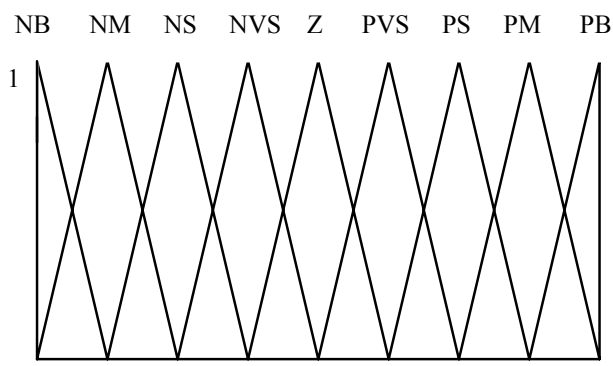

$$
\frac{-12 C}{100} \quad \frac{-9 C}{100} \frac{-6 C}{100} \quad \frac{-3 C}{100} \quad 0 \quad \frac{3 C}{100} \quad \frac{6 C}{100} \quad \frac{9 C}{100} \frac{12 C}{100}
$$

NB: Negative Big NM: Negative Middle NS: Negative Small NVS: Negative Very Small Z: Zero PVS: Positive Very Small PS: Positive Small PM: Positive Middle PB: Positive Big

Fig.5. Membership functions for tuning number of the guard channels

\section{Fuzzy Rule Base}

The Fuzzy Rule Base consists a series of 30 fuzzy rules, shown in Table 1. The control rules have the following form: IF "conditions", THEN "action". For example, if the CDP is
Small, and number of the guard channels is Very Small, then it triggers the $11^{\text {th }}$ rule and makes tuning number of the guard channels Positive Small.

Thus, fuzzy controller can compute the tuning number of the guard channels according to the CDP and current number of the guard channels. The fuzzified output parameter can be converted to a crisp value by the maximum membership inference method.

\begin{tabular}{|l|c|c|c|}
\multicolumn{4}{|c}{ Table 1 Fuzzy control rules } \\
\hline $\begin{array}{l}\text { Rule } \\
\text { Number }\end{array}$ & $\begin{array}{c}\text { IF } \\
\text { Call dropping } \\
\text { probability }\end{array}$ & $\begin{array}{c}\text { Aumber of the } \\
\text { guard channels }\end{array}$ & $\begin{array}{c}\text { THEN } \\
\text { Tuning number of the } \\
\text { guard channels }\end{array}$ \\
\hline R1 & Zero & Very Small & Zero \\
\hline R2 & Zero & Small & Negative Very Small \\
\hline R3 & Zero & Middle & Negative Small \\
\hline R4 & Zero & Big & Negative Middle \\
\hline R5 & Zero & Very Big & Negative Big \\
\hline R6 & Very Small & Very Small & Zero \\
\hline R7 & Very Small & Small & Zero \\
\hline R8 & Very Small & Middle & Zero \\
\hline R9 & Very Small & Big & Zero \\
\hline R10 & Very Small & Very Big & Zero \\
\hline R11 & Small & Very Small & Positive Small \\
\hline R12 & Small & Small & Positive Very Small \\
\hline R13 & Small & Middle & Zero \\
\hline R14 & Small & Big & Zero \\
\hline R15 & Small & Very Big & Zero \\
\hline R16 & Middle & Very Small & Positive Middle \\
\hline R17 & Middle & Small & Positive Small \\
\hline R18 & Middle & Middle & Positive Very Small \\
\hline R19 & Middle & Big & Positive Very Small \\
\hline R20 & Middle & Very Big & Zero \\
\hline R21 & Big & Very Small & Positive Big \\
\hline R22 & Big & Small & Positive Middle \\
\hline R23 & Big & Middle & Positive Small \\
\hline R24 & Big & Big & Positive Very Small \\
\hline R25 & Big & Very Big & Zero \\
\hline R26 & Very Big & Very Small & Positive Big \\
\hline R27 & Very Big & Small & Positive Middle \\
\hline R28 & Very Big & Middle & Positive Small \\
\hline R29 & Very Big & Big & Positive Very Small \\
\hline R30 & Very Big & Very Big & Zero \\
\hline
\end{tabular}

\section{Simulation}

\section{A. Simulation Parameters}

In order to evaluate the performance of our fuzzy scheme, we implement and simulate an adaptive channel reservation scheme [3] for comparison. To fairly contrast our scheme to the adaptive algorithm, we used the traffic model and parameters given in [3]. We assume total channel capacity of a cell is 50 . We also assume that the arrival processes of new call and handoff call are Poisson with mean arrival rates of $\lambda_{n}$ and $\lambda_{h}$ respectively. Channel holding times of both types of calls are assumed to follow a negative exponential distribution with mean $1 / \mu$. In the simulation, we set that 
$\lambda_{n} / \lambda_{h}=5 / 1$ and $1 / \mu=180$ seconds. The total simulation time is chosen to be 24 hours.

\section{B. Simulation Results}

The performance measures obtained through the simulation are the CDP, the $\mathrm{CBP}$ and channel utilization. We simulation when new call arrival rate changes from 10 calls per minute to 35 calls per minute. These performance measures are plotted as a function of the new call arrival rate.

Simulation curves of the CDP of the two schemes are shown in Fig.6. From the figure, we can see when traffic load changes, the values of the CDP of two schemes are lower than threshold 0.01 . When the traffic load is low (e.g., new call arrival rate equals to 10 calls/minute), the values of the CDP of two schemes are equal. As the traffic load increases, the CDP of the fuzzy scheme is lower than the adaptive scheme obviously. It indicates that the fuzzy scheme has a better robust performance. It can adapt to changes in the network load.

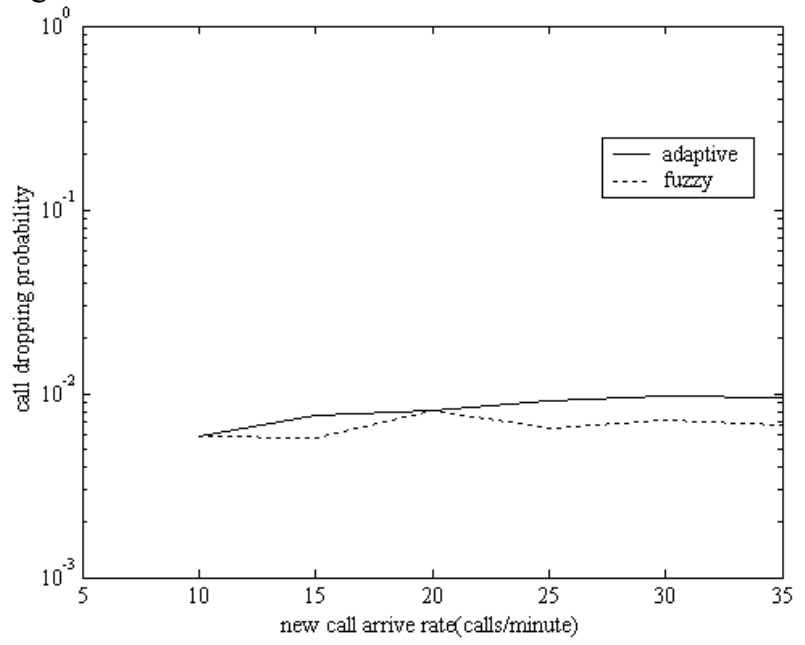

Fig.6. Call dropping probability

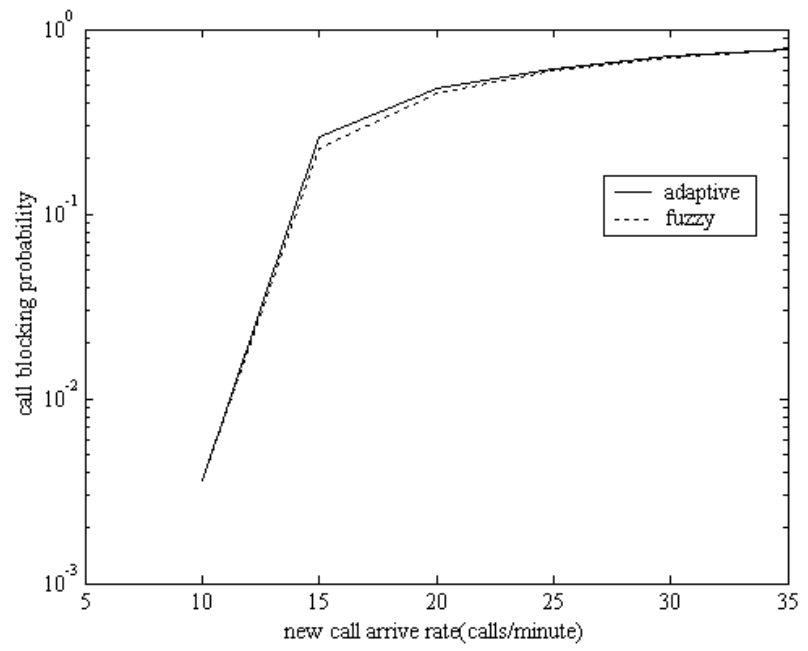

Fig.7. Call blocking probability

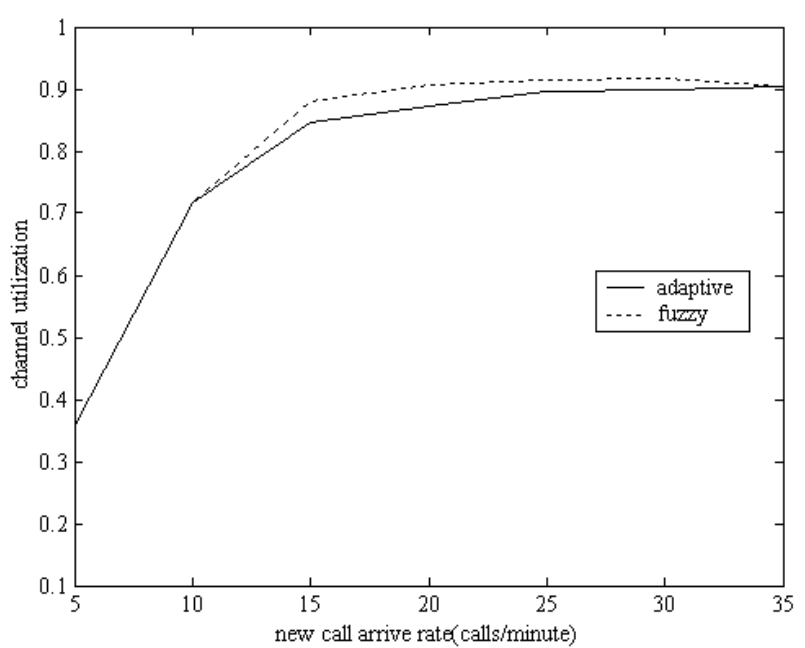

Fig.8. Channel utilization

Simulation curves of the CBP are shown in Fig.7. We can notice that the values of the CBP increase as the traffic load increases for both schemes. When the traffic load is low (e.g., new call arrival rate equals to 10 calls/minute), the values of the CBP of both schemes are equal. They both adjust $\mathrm{C}_{\mathrm{h}}$ to the minimum 0 to reduce the CBP. As the traffic load increases, the CBP of the fuzzy scheme is lower than the adaptive scheme. It indicates that our proposed algorithm can adjust $\mathrm{C}_{\mathrm{h}}$ better to reduce the CBP than the adaptive algorithm.

Simulation curves of the channel utilization are shown in Fig.8. We can see from the figure, channel utilization of the fuzzy scheme is higher than the adaptive scheme. It shows that the fuzzy scheme can utilize the resource more efficiently.

\section{CONCLUSION}

The paper presents an intelligent call admission control scheme based on fuzzy logic in wireless networks. It searches automatically the optimal number of the guard channels. Simulation results show the proposed scheme outperforms the adaptive channel reservation scheme. The fuzzy scheme has a better robust performance. It keeps the CDP and CBP low at the same time. It guarantees the QoS provision and increases the resource utilization of the network.

\section{REFERENCES}

[1] D. Hong and S. Rappaport, "Traffic Model and Performance Analysis for Cellular Mobile Radio Telephone Systems with Prioritized and Non-Prioritized Handoff Procedures," IEEE Trans. Vehicular Technology, vol.35, no.3, pp.77-92, 1986.

[2] Carlos Oliveira, Jaime Bae Kim, and Tatsuya Suda, "An Adaptive Bandwidth Reservation Scheme for High-Speed Multimedia Wireless Networks," IEEE Journal on Selected Areas in Communications, vol.16, no.6, pp. 858-874, Aug. 1998. 
[3] Yi Zhang and Derong Liu, "An Adaptive Algorithm for Call Admission Control in Wireless Network," in 2001 Proc. IEEE GLOBECOM Conf., pp.3628-3632.

[4] YouChang Ko, ChoongHo Cho, "Adaptive Handoff Guard Channel Allocation Scheme Using Fuzzy Logic in Personal Communications Service," in 1997 Proc. IEEE ICUPC Conf., pp.239-243.

[5] Sungwook Kim, Pramod K. Varshney, "An Adaptive Bandwidth Reservation Algorithm for Qos Sensitive Multimedia Cellular Networks," in 2002 Proc. IEEE Vehicular Technology Conf., pp.1475-1479.

[6] S.Boumerdassi, "An Efficient Reservation-based Dynamic Channel Assignment Strategy," in 2000 Proc. IEE $I^{\text {th }} 3 G$ Moblie Communication Technoligies Conf., pp.352-355.

[7] Theodore S. Rappaport, Wireless Communications Principles and Practice. New York: Prentice-Hall, 1996, ch. 2.

[8] Timothy J. Ross, Fuzzy Logic with Engineering Applications. New York: McGraw-Hill, 1995, ch. 13. 